\title{
Computational Analysis of Uremia Effects on Ventricular Action Potential
}

\author{
G Callisesi ${ }^{1}, \mathrm{C} \mathrm{Corsi}^{2}, \mathrm{~S} \mathrm{Severi}^{1}$ \\ ${ }^{1}$ University of Bologna, Cesena, Italy \\ ${ }^{2}$ University of Bologna, Bologna, Italy
}

\begin{abstract}
Patients suffering from renal failure often develop cardiac disturbances. In order to give a mathematical description of the effects of uremia on cardiac cellular excitability, the Ten Tusscher's model (2006) of human ventricular myocyte has been modified to incorporate the known effects of uremia on several ionic currents. The model was applied to study the effects of uremia and of the dialysis therapy on action potential duration (APD) and its restitution in response to the $S 1-S 2$ protocol. An altered transient repolarization and a shorter APD were found in the uremic myocyte. The APD restitution was also affected by uremia. At the end of dialysis, action potentials obtained after short diastolic intervals were characterized by the absence of the plateau phase. This could contribute to electrical inhomogeneity and explain the increase of arrhythmia occurrence often clinically observed in the last stage of the dialysis session.
\end{abstract}

\section{Introduction}

In recent years, studies on human myocytes have made available a large number of data on the gating properties of many channels, thus providing the information necessary for the development of in silico models of human action potential (AP). These models have been used to study several pathophysiological mechanisms linked, in particular, to the occurrence of cardiac arrhythmias. In this study such in silico approach have been applied to the analysis of the electrical activity of ventricular cells under conditions of uremia.

Patients suffering from renal failure often develop cardiac disturbances. More than $40 \%$ of the patients die prematurely because of cardiac complications $[1,2]$. The most common cardiac disease associated with renal failure is left ventricle hypertrophy accompanied by diastolic dysfunction $[3,4]$. Such uremic cardiomyopathy is linked to cardiac electrical and contractile dysfunction even at the single cell level. Albeit a number of factors have been implied in the pathogenesis of uremic cardiomyopathy the process is only partially understood.

Moreover, patients suffering from acute renal failure show an increased incidence of arrhythmic events. The origin of these alterations in myocardial electrical activity remains also poorly understood. The incidence of arrhythmic events increases especially during the hemodialysis sessions when, in order to restore a normal blood composition, the content of electrolytes in the extracellular fluid is significantly changed in a very short time interval.

Since the available computational models of ventricular cells don't include the known effects of uremic cardiomyopathy on electrical cell activity, the aim of the present study was to develop a model of an uremic ventricular cardiomyocyte in order to give a mathematical description of the effects of uremia on (1) the ionic currents, (2) the AP duration and morphology and (3) the action potential duration (APD) restitution.

In addition, the changes in ventricular electrical activity caused by hemodialysis have been analysed in this framework in order to suggest possible mechanisms responsible for the clinically observed repolarization alterations.

\section{Methods}

The Ten Tusscher's model (2006) of human epicardial ventricular myocyte (TTP06, Fig. 1) provided the basis for the AP simulations in this study. For a complete description of the model and its validation see [5]. Our modifications to the original formulation based on the available experimental data on the effects of uremia on several ionic currents are described below.

The maximal $\mathrm{I}_{\mathrm{NaK}}$ (Na-K pump current) was reduced by $10 \%$ according to several experimental data on red blood cells from uremic patients showing different degrees of functional deficit of the pump [6-11].

The maximal $\mathrm{I}_{\text {up }}$ (SERCA pump current) was reduced by $30 \%$ according to data from rat cardiac myocytes [12]. 


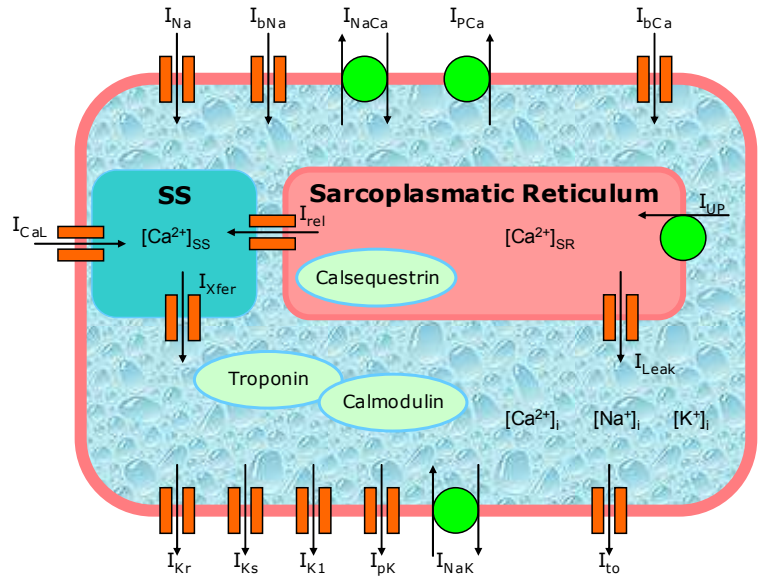

Figure 1. Schematic diagram of the Ten Tusscher ventricular cell model [5]. The model describes the main membrane currents and active transport mechanisms participating in the AP and the processes that regulate intracellular $\mathrm{Ca}^{2+}$ concentration, including dyadic space or subspace (SS) calcium dynamics that controls L-type $\mathrm{Ca}^{2+}$ current and $\mathrm{Ca}^{2+}$-induced $\mathrm{Ca}^{2+}$ release.

$\mathrm{Ca}^{2+}$-dependent inactivation and voltage-dependent inactivation time constants of $\mathrm{I}_{\mathrm{CaL}}\left(\mathrm{Ca}^{2+} \mathrm{L}\right.$-Type current) were both reduced by $20 \%$ according to experiments on rat ventricular cells [13].

The peak value of $I_{\text {to }}$ (transient outward potassium current) in conditions of uremia has been reported to be increased [14]. To reproduce this result, we increased the maximal conductance of the current from 294 to $400 \mathrm{~S} / \mathrm{F}$. Moreover we increased the time constant of inactivation, $\tau_{\mathrm{f}}$, by $2 \mathrm{~ms}$ at all the membrane potential values and modified the expression of the steady-state activation variable $r_{\infty}$ as follows:

$$
r_{\infty}=\frac{1}{1+e^{\frac{(10-V)}{6}} .}
$$

Pacing at $1 \mathrm{~Hz}$ was maintained until a steady AP was reached. APD was measured as the interval between the AP upstroke and the $90 \%$ repolarization level $\left(\mathrm{APD}_{90}\right)$. The APD restitution (APDR) was assessed by implementing the $\mathrm{S} 1-\mathrm{S} 2$ protocol. It consists of $10 \mathrm{~S} 1$ stimuli (at $1 \mathrm{~Hz}$ pacing rate) followed by a S2 extrastimulus delivered at some diastolic interval (DI) after the AP generated by the last S1 stimulus. The APDR curve is generated by decreasing DI and plotting APD corresponding to the S2 stimulus against DI.

The effects of dialysis on APD were simulated by imposing extracellular concentrations of the main electrolytes to the average values measured in vivo in a previous clinical study [15]. Before dialysis such values were (in mM): $1.14 \mathrm{Ca}^{2+}, 5.4 \mathrm{~K}^{+}$and $140 \mathrm{Na}^{+}$; whereas in the last stage of the dyalisis session they were (in $\mathrm{mM}$ ): $1.03 \mathrm{Ca}^{2+}, 3.7 \mathrm{~K}^{+}$and 143 .

Model differential equations were implemented in Simulink (Mathworks Inc., Natick, MA, U.S.A.). A variable order solver based on the numerical differentiation formulas (NDFs) was used to numerically solve the model equations (ode15s) [16 ].

\section{Results}

The AP generated by the model of uremic cardiomyocyte is different from the AP generated by the original TTP06 model in terms of duration and morphology (Fig. 2, thick line). In particular, transient repolarization (phase 1 of AP) is enhanced and APD is shorter in the uremic myocyte than in the normal one (277 ms vs $309 \mathrm{~ms}$ at $1 \mathrm{~Hz}$ ). Through simulation the effects of each modification to the TTP06 model has been analysed. Neither a reduction in $\mathrm{I}_{\mathrm{NaK}}$ nor in $\mathrm{I}_{\mathrm{up}}$ currents has significant effects on AP (curves superimposed to the TTP06 one in Fig. 2). As expected, modifications to $I_{\text {to }}$ lead to alteration of the phase 1 of AP (transient repolarization phase) and to AP shortening (Fig. 2, dashed line). Because of its role in sustaining the plateau, the main effect of an alterated $\mathrm{I}_{\mathrm{CaL}}$ current is the AP shortening (Fig. 2, dotted line).

The model predicts that the calcium transient is also affected, resulting significantly dumped, by uremia (Fig. 3 ). When simulated separately, modifications to $I_{\text {to }}$ and $\mathrm{I}_{\mathrm{NaK}}$ cause a rise in the calcium transient, while modifications to $\mathrm{I}_{\mathrm{CaL}}$ and $\mathrm{I}_{\text {up }}$ have the opposite effect. The overall result was a decrease of calcium transient (thick vs thin line in Fig. 3).

APDR was also affected by uremia, in fact, as shown

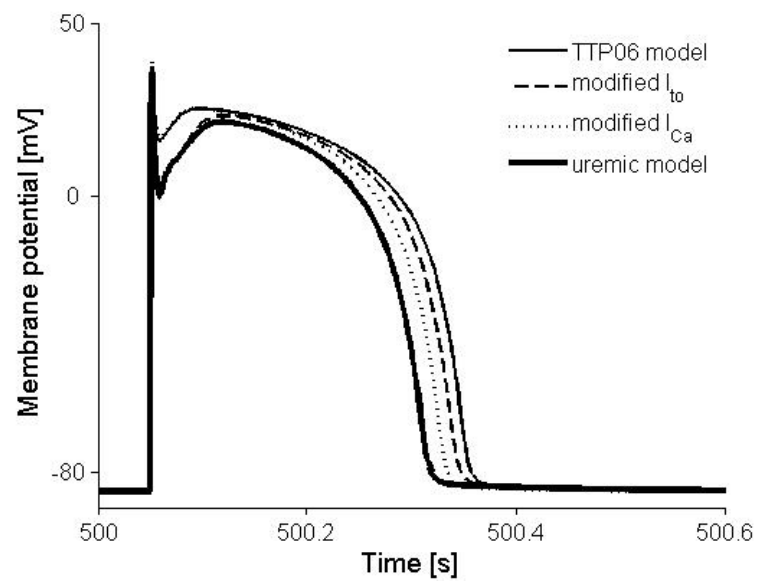

Figure 2. The APs generated by TTP06 model, by TTP06 model with modifications on $\mathrm{I}_{\text {to }}$ and $\mathrm{I}_{\mathrm{CaL}}$ only and by the uremic model. Uremia led to a shorter APD and an enhancement of transient repolarization (phase 1 of AP). 


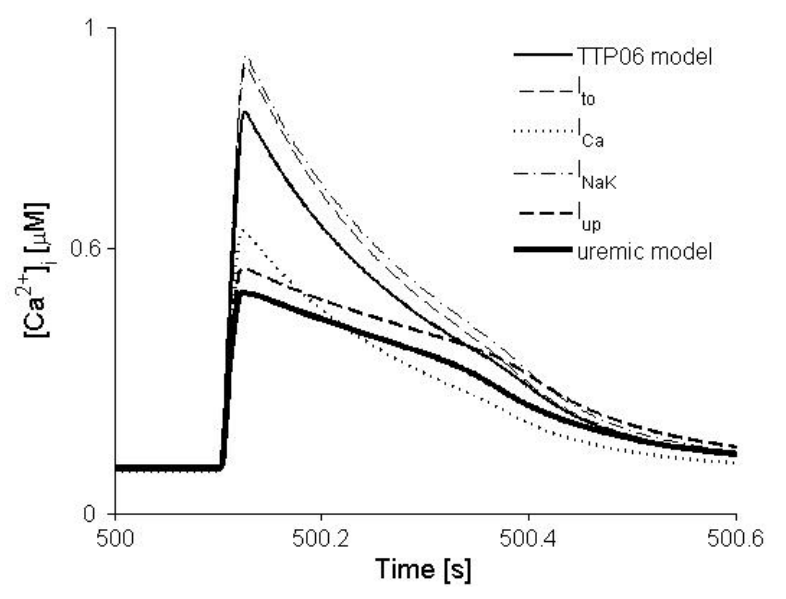

Figure 3. Intracellular $\mathrm{Ca}^{2+}$ transient generated by TTP06 model, by the uremic model and by modifications of single currents Uremia led to a dumped $\mathrm{Ca}^{2+}$ transient.

in Fig. 4, the restitution curve of the uremic AP has a decreased slope and is downshifted for DIs longer than $200 \mathrm{~ms}$.

The beginning and end dialysis conditions have been then analysed. At the end of dialysis the APDR curve is dramatically changed with respect to basal conditions (Fig. 5). In fact, APs obtained at short diastolic intervals are very short since they are characterized by the absence of the plateau phase (Fig. 6).

\section{Discussion and Conclusions}

In this study a model of uremic ventricular cardiomyocyte has been developed by acting on the

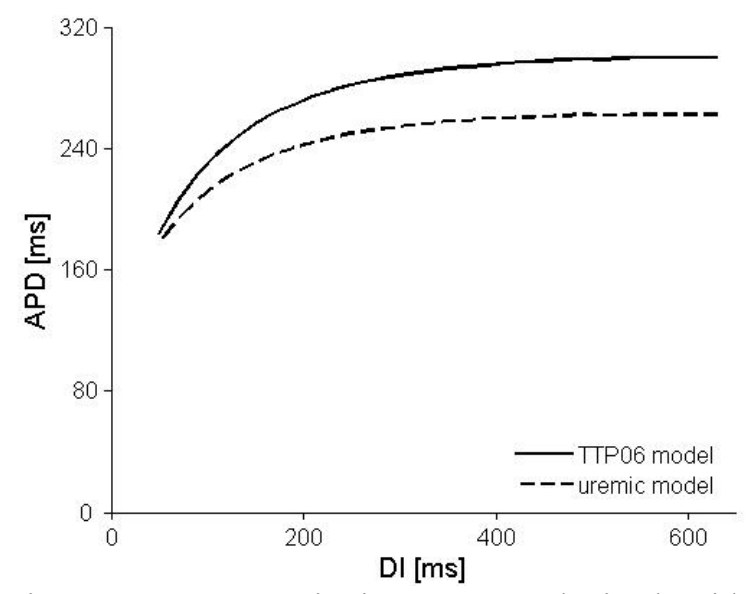

Figure 4. APD restitution curves obtained with the uremic and the TTP06 models (DI: diastolic interval, see Methods for a description of the S1-S2 protocol).

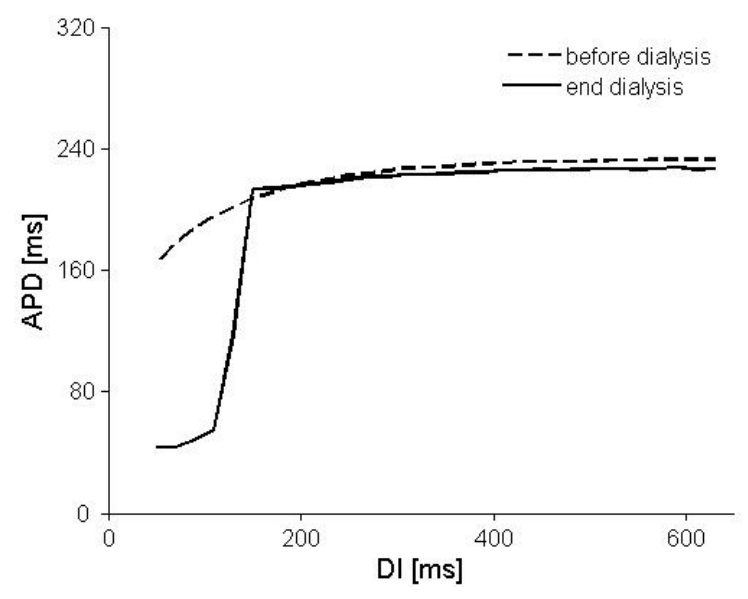

Figure 5. APD restitution curves obtained with the uremic model before and at the end of dialysis. The end dialysis APDR curve is dramatically changed with respect to basal conditions, showing a transition between very short APs for short DIs.

formulations of $\mathrm{I}_{\mathrm{to}}, \mathrm{I}_{\mathrm{CaL}}, \mathrm{I}_{\mathrm{up}}$ and $\mathrm{I}_{\mathrm{NaK}}$ currents and applied to analyse uremia effects on the action potential.

By means of the model all the information available at present from in vitro analysis of the uremia effects on ionic cardiac currents have been integrated and it has been possible to predict the global effects on the cardiac AP.

Model predictions indicated that uremia leads to

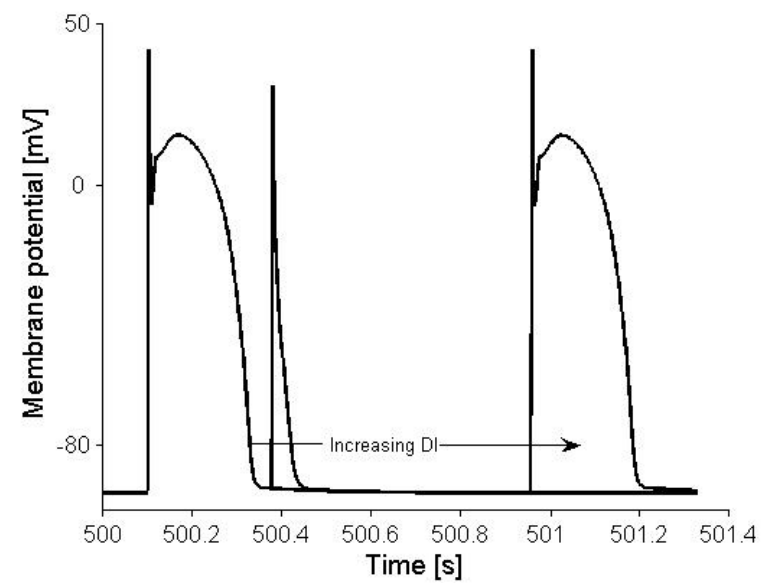

Fig. 6: AP waveforms from the restitution protocol in the end-dialysis condition. The last S1-induced AP is shown on the left together with two representative APs induced by S2 at different values of DI. After a long DI $(630 \mathrm{~ms})$ an AP similar to that induced by regular pacing at $1 \mathrm{~Hz}$ is elicited. After a short DI $(50 \mathrm{~ms})$ the absence of the AP plateau phase and a very short $\mathrm{AP}\left(\mathrm{APD}_{90}: 43.5 \mathrm{~ms}\right)$ can be observed. 
shortening of AP, because of modifications to $\mathrm{I}_{\text {to }}$ and $\mathrm{I}_{\mathrm{CaL}}$, and to dumped calcium transient, because of modifications to $\mathrm{I}_{\mathrm{CaL}}, \mathrm{I}_{\mathrm{up}}$. While the latter effect is coherent with a reduced contractility, which can be observed as clinical manifestation of cardiomyopathy, the interpretation of the former one is less clear. In fact, even if there is no consensus about the effects of uremia on the QT interval, longer QT than normal have been often reported in uremic patients suggesting a prolongation of APD at the cellular level. One possible explanation could be that macroscopic QT prolongation is more an effect of conduction slowing in hypertrophic hearts than APD prolongation at the single cell level.

Moreover, at the end of dialysis APs obtained at short diastolic intervals are very short and characterized by the absence of the plateau phase. Since this phenomenon was not observed in end-dialysis simulations with the original Ten Tusscher's model (not shown) our analysis suggests it as a uremia effect. This could contribute to electrical inhomogeneity in the last stage of the dialysis session, when an increase of arrhythmia occurrence is often clinically observed [17].

In conclusion, the present study can be considered only the first step towards the development of a mathematical model of the uremic cardiomyocyte since many other biochemical processes are involved in the pathological impact and in the compensatory cardiac response to uremia. The availability of a reliable model of uremic cardiomyocyte will be useful in order to study the mechanisms of the uremic cardiomiopathy and the risk of arrhythmic phenomena in dialysis patients.

\section{Acknowledgements}

The work was partially supported by Hospal SpA (Bologna, Italy).

\section{References}

[1] Raine AE, Margreiter R, Brunner FP, Ehrich JH, Geerlings W, Landais $P$ et al. Report on management of renal failure in Europe, XXII, 1991. Nephrol Dial Transplant 1992; 7 Suppl 2:7-35.

[2] Bleyer AJ, Hartman J, Brannon PC, Reeves-Daniel A, Satko SG, Russell G. Characteristics of sudden death in hemodialysis patients. Kidney Int 2006; 69(12):2268-2273.

[3] Clyne N, Lins LE, Pehrsson SK. Occurrence and significance of heart disease in uraemia. An autopsy study. Scand J Urol Nephrol 1986; 20(4):307-311.

[4] Lai KN, Ng J, Whitford J, Buttfield I, Fassett RG, Mathew TH. Left ventricular function in uremia: echocardiographic and radionuclide assessment in patients on maintenance hemodialysis. Clin Nephrol 1985; 23(3):125-133.
[5] Ten Tusscher KHWJ, Panfilov AV. Alternans and spiral breakup in a human ventricular tissue model. Am J Physiol Heart Circ Physiol 2006; 291(3):H1088-H1100.

[6] Corry DB, Lee DB, Tuck ML. A kinetic study of cation transport in erythrocytes from uremic patients. Kidney Int 1987; 32(2):256-260.

[7] Izumo H, Izumo S, DeLuise M, Flier JS. Erythrocyte Na,K pump in uremia. Acute correction of a transport defect by hemodialysis. J Clin Invest 1984; 74(2):581-588.

[8] Fervenza FC, Hendry BM, Ellory JC. Effects of dialysis and transplantation on red cell $\mathrm{Na}$ pump function in renal failure. Nephron 1989; 53(2):121-128.

[9] Prasad R, Mond R, Jain S, Kaur G, Chugh KS. Modulation of ouabain sensitive sodium potassium pump of erythrocytes from patients with chronic renal failure: role of acute hemodialysis. Biochem Mol Biol Int 1996; 40(6):1087-1094.

[10] Kaji D, Thomas K. Na+-K+ pump in chronic renal failure. Am J Physiol Renal Physiol 1987; 252(5):F785-F793.

[11] Welt LG, Sachs JR, Mcmanus TJ. An ion transport defect in erythrocytes from uremic patients. Trans Assoc Am Physicians 1964; 77:169-181.

[12] Kennedy D, Omran E, Periyasamy SM, Nadoor J, Priyadarshi A, Willey JC et al. Effect of Chronic Renal Failure on Cardiac Contractile Function, Calcium Cycling, and Gene Expression of Proteins Important for Calcium Homeostasis in the Rat. J Am Soc Nephrol 2003; 14(1):9097.

[13] Donohoe P, McMahon AC, Walgama OV, Bertaso F, Dockrell MEC, Cramp HA et al. L-type calcium current of isolated rat cardiac myocytes in experimental uraemia. Nephrol Dial Transplant 2000; 15(6):791-798.

[14] Donohoe P, Hendry BM, Walgama OV, Bertaso F, Hopster DJ, Shattock MJ et al. An Altered Repolarizing Potassium Current in Rat Cardiac Myocytes after Subtotal Nephrectomy. J Am Soc Nephrol 2000; 11(9):1589-1599.

[15] Severi S, Grandi E, Pes C, Badiali F, Grandi F, Santoro A. Calcium and potassium changes during haemodialysis alter ventricular repolarization duration: in vivo and in silico analysis. Nephrol Dial Transplant 2008; 23(4):1378-1386.

[16] Shampine LF, Reichelt MW. The MATLAB ODE Suite. SIAM Journal on Scientific Computing 1997;18:1-22.

[17] Santoro A, Mancini E, London G, Mercadal L, Fessy H, Perrone B et al. Patients with complex arrhythmias during and after haemodialysis suffer from different regimens of potassium removal. Nephrol Dial Transplant 2008; 23(4):1415-1421.

Address for correspondence

Stefano Severi

Biomedical Engineering Laboratory - D.E.I.S., University of Bologna

Via Venezia 52, I-47023 Cesena - ITALY

E-mail: stefano.severi@unibo.it 